ITP-UU-11/29, SPIN-11/22

CCTP-2011-22, UFIFT-QG-11-06

\title{
Gauging away Physics
}

\author{
S. P. Miao* \\ Institute for Theoretical Physics 83 Spinoza Institute, Utrecht University \\ Leuvenlaan 4, Postbus 80.195, 3508 TD Utrecht, NETHERLANDS \\ N. C. Tsamis ${ }^{\dagger}$ \\ Institute of Theoretical Physics 85 Computational Physics, and \\ Department of Physics, University of Crete \\ GR-710 03 Heraklion, HELLAS \\ R. P. Woodard ${ }^{\ddagger}$ \\ Department of Physics, University of Florida \\ Gainesville, FL 32611, UNITED STATES
}

\begin{abstract}
We consider the recent argument by Higuchi, Marolf and Morrison [1] that a nonlocal gauge transformation can be used to eliminate the infrared divergence of the graviton propagator, when evaluated in Bunch-Davies vacuum on the open coordinate submanifold of de Sitter space in transverse-tracelesssynchronous gauge. Because the transformation is not local, the equal time commutator of undifferentiated fields no longer vanishes. From explicit examination of the Wightman function we demonstrate that the transformation adds anti-sources in the far future which cancel the bad infrared behavior but also change the propagator equation. The same problem exists in the localized version of the recent argument. Adding such anti-sources does not seem to be legitimate and could be used to eliminate the infrared divergence of the massless, minimally coupled scalar. The addition of such anti-sources in flat space QED could be effected by an almost identical gauge transformation, and would seem to eliminate the well known infrared divergences which occur in loop corrections to exclusive amplitudes.
\end{abstract}

PACS numbers: 04.62.+v, 04.60-m, 98.80.Cq

* e-mail: S.Miao@uu.nl

${ }^{\dagger}$ e-mail: tsamis@physics.uoc.gr

‡ e-mail: woodard@phys.ufl.edu 


\section{Introduction}

There is a wide disparity of opinion concerning quantum field theory in the de Sitter geometry between those who approach the subject from the perspective of cosmology and those who view it from the perspective of mathematical physics. Cosmologists tend to see the de Sitter geometry as an idealized special case of the larger class of spatially flat, Friedman-Robertson-Walker (FRW) geometries, whose invariant element in conformal coordinates is,

$$
d s_{\mathrm{FRW}}^{2}=a^{2}(\eta)\left[-d \eta^{2}+d \vec{x} \cdot d \vec{x}\right] \quad \text { de Sitter } \Longrightarrow a(\eta)=-\frac{\ell}{\eta} .
$$

Many mathematical physicists find this view antipathetic. To them de Sitter has a privileged status as the unique, maximally symmetric solution to the Einstein equations with positive cosmological constant $\left(\Lambda=(d-1) / \ell^{2}\right.$ in $d$ spacetime dimensions), and the explication of its properties requires the use of coordinates which cover the full de Sitter manifold.

The matter of coordinate symmetries illustrates the contrasting perspectives. The key symmetries for cosmologists are homogeneity and isotropy,

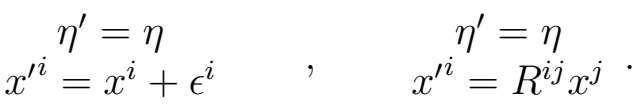

Mathematical physicists attach equal importance to dilatations and spatial special conformal transformations,

$$
\begin{array}{rlrl}
\eta^{\prime} & =k \eta \\
x^{\prime i} & =k x^{i} & \eta^{\prime} & =\frac{\eta}{1-2 \vec{\theta} \cdot \vec{x}+\theta^{2} x \cdot x}
\end{array},
$$

where $x \cdot x \equiv-\eta^{2}+\vec{x} \cdot \vec{x}$ and $\theta^{2} \equiv \vec{\theta} \cdot \vec{\theta}$. The union of (2) and (3) comprises the full de Sitter group, and mathematical physicists typically expect that it should play the same role in organizing quantum field theory on de Sitter space as the Poincaré group does for Minkowski space. Cosmologists are not especially concerned with (3) because their interest in de Sitter is as an approximation to primordial inflation, during which the slight time dependence of the Hubble parameter breaks these symmetries.

For most quantum fields there is not much practical difference between the two viewpoints. Fields with positive mass-squared, and massless, conformally invariant fields admit a de Sitter invariant formulation. However, 
even as it is for flat space, one gets the correct answer by following canonical quantization, without taking advantage of coordinate symmetries. The fields which occasion conflict are those which are both massless and not conformally invariant: massless, minimally coupled (MMC) scalars and the graviton. These are the fields responsible for primordial perturbations in the theory of inflation [2]. In the de Sitter limit, the unique de Sitter invariant wave function for these fields is Bunch-Davies vacuum [3]. The scalar and tensor power spectra are defined in this vacuum, and it is obvious from their scale invariance (which becomes exact in the de Sitter limit) that the Fourier mode sums for the propagators of both fields must diverge in the infrared [4]. What to make of this has provoked decades of controversy.

For MMC scalars there is general agreement that the infrared divergence means the Bunch-Davies wave function is not normalizable and hence not a state. The de Sitter problem can be viewed as but a special case of the infrared divergences discovered in 1977 by Ford and Parker [5] for cosmologies with deceleration parameter less than or equal to the matter-dominated value of $q=+\frac{1}{2}$. (de Sitter has $q=-1$, and data constrains single-scalar inflation to the range $-1<q<-0.986$ [4].) Infrared divergences signal something unphysical about the question being posed. For example, the infrared divergences in loop corrections to the exclusive amplitudes of flat space quantum electrodynamics (QED) arise because no real experiment is able to exclude the possibility of a small amount of energy escaping detection in the form of very soft photons. When one computes inclusive rates and cross sections the results are infrared finite but they display a measured dependence upon the detector sensitivity [6]. For cosmologies with $q \leq+\frac{1}{2}$ the problem is that one cannot measure the super-horizon modes to be in perfect Bunch-Davies vacuum, or anything else. Once the assumption of Bunch-Davies vacuum is relaxed for the super-horizon modes [7] one gets finite propagators which, however, show secular growth [8].

It will be noted that the infrared problem of MMC scalars in de Sitter rests comfortably within the larger context of spatially flat, FRW geometries, just as cosmologists prefer. That does not mean there has been no special attention to de Sitter. A famous result was derived in 1982 for the secular — and de Sitter breaking — growth of the coincidence limit of the MMC scalar propagator by Vilenkin and Ford, by Linde and by Starobinsky [9]. Mathematical physicists were finally persuaded of the reality of de Sitter breaking for this system by the formal proof Allen and Folacci presented in 1987 [10]. However, there has been a notable tendency among the math- 
ematically inclined to discount de Sitter breaking because the expectation value of the free stress tensor is de Sitter invariant. That is the result of derivatives in the free stress tensor, and merely postpones the appearance of secular, de Sitter breaking effects to loop corrections in any theory which possesses nonderivative interactions [11, 12, 13].

No similar consensus has been reached for gravitons, which we define as the perturbation of the full metric from its background value,

$$
h_{\mu \nu}(\eta, \vec{x}) \equiv \frac{1}{\sqrt{32 \pi G}}\left[g_{\mu \nu}(\eta, \vec{x})-a^{2}(\eta) \eta_{\mu \nu}\right] \equiv a^{2}(\eta) \widetilde{h}_{\mu \nu}(\eta, \vec{x})
$$

Because we shall have no more need for the full metric, we henceforth employ the symbol $g_{\mu \nu}(\eta, \vec{x})$ to describe the background metric, $g_{\mu \nu}(\eta, \vec{x}) \equiv a^{2}(\eta) \eta_{\mu \nu}$. Gravitons are closely related to MMC scalars because Grishchuk [14] long ago showed that conformally rescaled graviton fields which are purely spatial, transverse and traceless,

$$
\widetilde{h}_{\mu 0}(\eta, \vec{x})=0 \quad, \quad \partial_{i} \widetilde{h}_{i j}(\eta, \vec{x})=0 \quad, \quad \widetilde{h}_{i i}(\eta, \vec{x})=0,
$$

obey exactly the same linearized equation of motion as the MMS scalar,

$$
\partial_{\mu}\left[\sqrt{-g} g^{\mu \nu} \partial_{\nu} h_{i j}(\eta, \vec{x})\right] \equiv \sqrt{-g} \square \widetilde{h}_{i j}(\eta, \vec{x})=0 \text {. }
$$

(Note that we really do mean $\square$ to be the covariant scalar d'Alembertian, even though it acts on a tensor field.) Just as in flat space, one can prove that there are no other dynamical gravitons by first solving the full linearized field equations in some volume gauge condition — for example, de Donder gauge - and then imposing a residual gauge condition on the solutions [15].

Grishchuk's result (6) means that dynamical gravitons must suffer from the same infrared problems as MMC scalars, not just in de Sitter but for any spatially flat, FRW cosmology with $q \leq+\frac{1}{2}$. This can be seen by studying any one of the standard 2-point functions: the retarded Green's function, the Wightman function or the Feynman propagator,

$$
\begin{aligned}
{\left[{ }_{i j} G_{k \ell}\right]\left(x ; x^{\prime}\right) } & \equiv-i \theta\left(\eta-\eta^{\prime}\right)\left[\widetilde{h}_{i j}(\eta, \vec{x}), \widetilde{h}_{k \ell}\left(\eta^{\prime}, \vec{x}^{\prime}\right)\right], \\
{\left[{ }_{i j} W_{k \ell}\right]\left(x ; x^{\prime}\right) } & \equiv\left\langle\Omega\left|\widetilde{h}_{i j}(\eta, \vec{x}) \widetilde{h}_{k \ell}\left(\eta^{\prime}, \vec{x}^{\prime}\right)\right| \Omega\right\rangle \\
\left.i{ }_{i j} \Delta_{k \ell}\right]\left(x ; x^{\prime}\right) & \equiv\left\langle\Omega\left|T\left[\widetilde{h}_{i j}(\eta, \vec{x}) \widetilde{h}_{k \ell}\left(\eta^{\prime}, \vec{x}^{\prime}\right)\right]\right| \Omega\right\rangle .
\end{aligned}
$$

(Here $|\Omega\rangle$ stands for Bunch-Davies vacuum and $T$ denotes the time-ordering symbol.) Although the mode sum for the retarded Green's function exists, 
its properties imply infrared divergences for the Wightman function and for the propagator, which is confirmed by direct examination of the mode sums for these quantities.

Before proceeding it is useful to digress on the properties of the three 2-point functions. Each of them obeys a simple equation when acted upon by the scalar d'Alembertian,

$$
\begin{aligned}
\sqrt{-g} \square\left[{ }_{i j} G_{k \ell}\right]\left(x ; x^{\prime}\right) & =\left[\Pi_{i(k} \Pi_{\ell) j}-\frac{\Pi_{i j} \Pi_{k \ell}}{d-2}\right] \delta^{d}\left(x-x^{\prime}\right), \\
\sqrt{-g} \square\left[{ }_{i j} W_{k \ell}\right]\left(x ; x^{\prime}\right) & =0, \\
\sqrt{-g} \square i\left[{ }_{i j} \Delta_{k \ell}\right]\left(x ; x^{\prime}\right) & =\left[\Pi_{i(k} \Pi_{\ell) j}-\frac{\Pi_{i j} \Pi_{k \ell}}{d-2}\right] i \delta^{d}\left(x-x^{\prime}\right) ;
\end{aligned}
$$

where the transverse projection operator $\Pi_{i j}$ is defined by Fourier transform just as in flat space,

$$
\Pi_{i j} \equiv \delta_{i j}-\frac{\partial_{i} \partial_{j}}{\partial_{k} \partial_{k}}
$$

To prevent indices (and the transverse-traceless projection operator) from becoming an issue, we contract them across the $x^{\mu}$ and $x^{\prime \mu}$ index groups,

$$
\left.G\left(x ; x^{\prime}\right) \equiv{ }_{i j} G_{i j}\right]\left(x ; x^{\prime}\right), W\left(x ; x^{\prime}\right) \equiv\left[{ }_{i j} W_{i j}\right]\left(x ; x^{\prime}\right), i \Delta\left(x ; x^{\prime}\right) \equiv i\left[{ }_{i j} \Delta_{i j}\right]\left(x ; x^{\prime}\right) \text {. }
$$

One then sees that these contracted graviton 2-point functions obey precisely the same equations as $\frac{1}{2} d(d-3)$ times the analogous MMC scalar 2-point function, which are $\sqrt{-g} \square W\left(x ; x^{\prime}\right)=0$ and,

$$
i \sqrt{-g} \square G\left(x ; x^{\prime}\right)=\frac{1}{2} d(d-3) i \delta^{d}\left(x-x^{\prime}\right)=\sqrt{-g} \square i \Delta\left(x ; x^{\prime}\right) .
$$

Of course this is because each of the $\frac{1}{2} d(d-3)$ graviton polarizations behaves like an independent MMC scalar.

Grishchuk's result poses a terrible obstacle for those who believe that a de Sitter invariant state (as opposed to a possibly non-normalizable wave function) exists for dynamical gravitons. Although the unique solution for $G\left(x ; x^{\prime}\right)$ follows from classical general relativity and is de Sitter invariant, it cannot be analytically continued to give either a de Sitter invariant Wightman function or a de Sitter invariant propagator. To see this, first compare expressions (7) and (91) to conclude,

$$
G\left(x ; x^{\prime}\right)=2 \theta\left(\eta-\eta^{\prime}\right) \operatorname{Im}\left[i \Delta\left(x ; x^{\prime}\right)\right] .
$$


In $d=4$ dimensions the retarded Green's function is [16],

$$
\lim _{d \rightarrow 4} \frac{G\left(x ; x^{\prime}\right)}{\frac{1}{2} d(d-3)}=-\frac{\theta\left(\eta-\eta^{\prime}\right)}{8 \pi \ell^{2}}\left\{\delta\left(z\left(x ; x^{\prime}\right)-1\right)+2 \theta\left(z\left(x ; x^{\prime}\right)-1\right)\right\},
$$

where $1-z\left(x ; x^{\prime}\right) \equiv \frac{1}{4} a(\eta) a\left(\eta^{\prime}\right) \ell^{-2}\left(x-x^{\prime}\right)^{\mu}\left(x-x^{\prime}\right)^{\nu} \eta_{\mu \nu}$ is a de Sitter invariant function of the proper length from $x^{\mu}$ to $x^{\prime \mu}$. It is possible to get both the delta function and the theta function from simple analytic continuations,

$$
\delta(z-1)=-\frac{1}{\pi} \operatorname{Im}\left[\frac{1}{1-z+i \epsilon}\right] \quad, \quad \theta(z-1)=\frac{1}{\pi} \operatorname{Im}[\ln (1-z+i \epsilon)] .
$$

However, the resulting function does not obey the propagator equation (15). The most general solution consistent with homogeneity and isotropy takes the form [16],

$$
\lim _{d \rightarrow 4} \frac{i \Delta\left(x ; x^{\prime}\right)}{\frac{1}{2} d(d-3)}=\frac{1}{16 \pi^{2} \ell^{2}}\left\{\frac{1}{1-z\left(x ; x^{\prime}\right)+i \epsilon}-2 \ln \left[\frac{1-z\left(x ; x^{\prime}\right)+i \epsilon}{a(\eta) a\left(\eta^{\prime}\right)}\right]+\text { Const. }\right\} .
$$

The explicit dependence of (19) upon the scale factors is an example of the secular behavior mentioned previously. This kind of secular dependence has nothing special to do with de Sitter and is a feature of all spatially flat, FRW cosmologies with $q \leq+\frac{1}{2}[8]$. Note also that the de Sitter breaking part of (19) drops out from the mixed second derivative $\partial_{\mu} \partial_{\nu}^{\prime} i \Delta\left(x ; x^{\prime}\right)$, which is why the expectation value of the MMC scalar stress tensor is de Sitter invariant, even though the propagator is not. The same thing occurs for the tree order Weyl-Weyl correlator and in no way implies that dynamical gravitons are de Sitter invariant.

The argumentation just reviewed would seem decisive, but mathematical physicists have for years ignored it because they were able to derive manifestly de Sitter invariant expressions for the graviton propagator by adding covariant gauge fixing terms to the Euclidean action and then analytically continuing [17]. It was recently shown that both procedures are invalid, the first because it adds spurious zero modes [18] and the second because it automatically subtracts power law infrared divergences [19]. Further, an explicitly de Sitter breaking result for the propagator has been derived in a covariant exact gauge without subtracting infrared divergences [20].

A sort of compromise has recently been suggested, based on some old work by Higuchi [21]. The idea is that dynamical gravitons on de Sitter might be 
physically de Sitter invariant, even though no manifestly de Sitter invariant propagator for them can be found [22]. This proposal entails making sense of Bunch-Davies vacuum, which is the unique possibility for a de Sitter invariant state for dynamical gravitons, and that means somehow avoiding the infrared divergence. Recall that the scale invariance of the graviton power spectrum, which becomes exact in the de Sitter limit, implies infrared divergences in the mode sums for both the Bunch-Davies Wightman function and for the Bunch-Davies propagator [4].

It does not seem possible to argue that the scale invariance of the graviton power spectrum is a gauge artifact. This is not only an observable quantity but one which the Planck satellite is even now attempting to observe. Nevertheless, Higuchi, Marolf and Morrison have recently devised an ingenious argument that the infrared divergences of the mode sums for $W\left(x ; x^{\prime}\right)$ and $i \Delta\left(x ; x^{\prime}\right)$ can be eliminated by making what seems to be a gauge transformation which preserves the transverse-traceless-synchronous conditions (5) [1. The purpose of this paper is to critically examine that argument, which we review in section 2 . In section 3 we demonstrate that the transformation changes both the propagator equation and the canonical commutation relations. We also show that the same procedure could be used to avoid the conclusion that no de Sitter invariant state exists for the MMC scalar. A very similar gauge transformation even seems to eliminate the infrared divergences of flat space QED. Our discussion comprises section 4.

\section{The Transformation}

With one major exception, we have followed the notation of Higuchi, Marolf and Morrison [1], except for correcting a few typoes, rearranging some factors and making the spacetime dependence of the mode functions explicit. The major notational change is that we use a tilde to denote conformally rescaled fields whereas they employ it to represent their transformed fields. We use a prime to indicate transformed fields.

The mode functions for a graviton with wave vector $\vec{k}$ and spin $s$ take the form,

$$
\gamma_{i j}^{s}(\eta, \vec{x} ; \vec{k})=a^{2}(\eta) \times \frac{N \sqrt{\ell}}{a^{\frac{d-1}{2}}} H_{\frac{d-1}{2}}^{(2)}(k \eta) e^{i \vec{k} \cdot \vec{x}} \times \epsilon_{i j}^{s}(\vec{k}) \equiv a^{2}(\eta) \widetilde{\gamma}_{i j}^{s}(\eta, \vec{x} ; \vec{k})
$$


Here the normalization constant is,

$$
N \sqrt{\ell} \equiv \bar{N}=\sqrt{\frac{\ell \pi}{4(2 \pi)^{d-1}}}
$$

and the transverse-traceless polarization tensors are the same as those of flat space,

$$
k_{i} \epsilon_{i j}^{s}(\vec{k})=0=\epsilon_{i i}^{s}(\vec{k}) \quad, \quad \epsilon_{i j}^{r}(\vec{k}) \epsilon_{i j}^{s *}(\vec{k})=\delta^{r s} .
$$

It is worth noting that the central term in expression (20) is the mode function for a massless, minimally coupled scalar with wave vector $\vec{k}$,

$$
u(\eta, \vec{x} ; \vec{k})=\frac{\bar{N}}{a^{\frac{d-1}{2}}} H_{\frac{d-1}{2}}^{(2)}(k \eta) e^{i \vec{k} \cdot \vec{x}}=\frac{i \bar{N}}{\pi} \Gamma\left(\frac{d-1}{2}\right)\left(\frac{2}{\ell k}\right)^{\frac{d-1}{2}}\left\{1+O\left(k^{2} \eta^{2}\right)\right\} e^{i \vec{k} \cdot \vec{x}}
$$

Mode functions are $\mathbb{C}$-number solutions of the linearized field equations. The operator which annihilates a graviton with wave number $\vec{k}$ and spin $s$ is $a_{s}(\vec{k})$. The nonzero part of the commutation algebra is,

$$
\left[a_{r}(\vec{k}), a_{s}^{\dagger}(\vec{p})\right]=\delta^{r s} \delta^{d-1}(\vec{k}-\vec{p}) .
$$

The mode sum for the transverse-traceless-synchronous graviton field operator is,

$$
h_{i j}(\eta, \vec{x})=a^{2}(\eta) \int d^{d-1} k \sum_{s}\left\{\widetilde{\gamma}_{i j}^{s}(\eta, \vec{x} ; \vec{k}) a_{s}(\vec{k})+\widetilde{\gamma}_{i j}^{s *}(\eta, \vec{x} ; \vec{k}) a_{s}^{\dagger}(\vec{k})\right\} .
$$

Note that raising one index with the full metric gives the conformally rescaled graviton field, whose indices are raised and lowered with $\delta_{i j}$,

$$
h_{j}^{i}(\eta, \vec{x}) \equiv g^{i k} h_{k j}(\eta, \vec{x})=\widetilde{h}_{i j}(\eta, \vec{x})
$$

The full equal-time commutation algebra of the fields is,

$$
\begin{aligned}
{\left[\widetilde{h}_{i j}(\eta, \vec{x}), \widetilde{h}_{k \ell}(\eta, \vec{y})\right] } & =0=\left[\partial_{0} \widetilde{h}_{i j}(\eta, \vec{x}), \partial_{0} \widetilde{h}_{k \ell}(\eta, \vec{y})\right] \\
{\left[\widetilde{h}_{i j}(\eta, \vec{x}), \partial_{0} \widetilde{h}_{k \ell}(\eta, \vec{y})\right] } & =\left[\Pi_{i(k} \Pi_{\ell) j}-\frac{\Pi_{i j} \Pi_{k \ell}}{d-2}\right] \frac{i \delta^{d-1}(\vec{x}-\vec{y})}{a^{d-2}} .
\end{aligned}
$$

Of course fields are the primary objects of quantum field theory so one really starts with the field equation (6) for $\widetilde{h}_{i j}(\eta, \vec{x})$. That implies the form (25) 
with relations (20-22). The Fourier coefficients $a_{s}(\vec{k})$ and $a_{s}^{\dagger}(\vec{k})$ of each mode function are properly combinations of the initial values of the field and its first time derivative. The equal-time commutation relations (27, 28) of these fields imply (24).

The unique de Sitter invariant wave function for this field is Bunch-Davies vacuum defined by the condition,

$$
a_{s}(\vec{k})|\Omega\rangle=0 \quad \forall \vec{k} \text { and } s .
$$

If we incorrectly assume $\langle\Omega \mid \Omega\rangle=1$ it is simple to show that the contracted graviton Wightman function defined by expressions (8) and (14) is just $\frac{1}{2} d(d-$ 3) copies of the Wightman function for a massless, minimally coupled scalar,

$$
\begin{aligned}
W\left(x ; x^{\prime}\right) & =\int d^{d-1} k \sum_{s} \widetilde{\gamma}_{i j}^{s}(\eta, \vec{x} ; \vec{k}) \widetilde{\gamma}_{i j}^{* *}\left(\eta^{\prime}, \vec{x}^{\prime} ; \vec{k}\right) \\
& =\frac{1}{2} d(d-3) \int d^{d-1} k u(\eta, \vec{x} ; \vec{k}) u^{*}\left(\eta^{\prime}, \vec{x}^{\prime} ; \vec{k}\right) .
\end{aligned}
$$

Owing to the small $k$ behavior of the scalar mode functions (23), expression (31) fails to converge in the infrared. The usual interpretation is that the single assumption from which these relations were derived $-\langle\Omega \mid \Omega\rangle=1$ - is false and, like the massless, minimally coupled scalar, there are no normalizable, de Sitter invariant states for the graviton.

Higuchi, Marolf and Morrison have argued that this conclusion is wrong [1. They assert that the infrared divergence of (30,31) is pure gauge and can be eliminated by what seems to be a linearized gauge transformation. The transformation parameters are $\xi_{0}(\eta, \vec{x})=0$ and,

$$
\xi_{i}(\eta, \vec{x})=-a^{2}(\eta) \int d^{d-1} k e^{-\rho k} \sum_{s}\left\{\widetilde{\gamma}_{i j}^{s}(0, \overrightarrow{0} ; \vec{k}) a_{s}(\vec{k})+\widetilde{\gamma}_{i j}^{s *}(0, \overrightarrow{0} ; \vec{k}) a_{s}^{\dagger}(\vec{k})\right\} x^{i},
$$

where $\rho$ is some positive constant. The transformed graviton field is,

$$
h_{\mu \nu}^{\prime}(\eta, \vec{x}) \equiv h_{\mu \nu}(\eta, \vec{x})+\nabla_{(\mu} \xi_{\nu)}(\eta, \vec{x}) .
$$

The new fields also obey the transverse-traceless-synchronous conditions (5)). The mode sums for their nonzero components are,

$$
\begin{aligned}
\widetilde{h}_{i j}^{\prime}(\eta, \vec{x})=\int d^{d-1} k \sum_{s}\left\{\left[\widetilde{\gamma}_{i j}^{s}(\eta, \vec{x} ; \vec{k})-e^{-\rho k} \widetilde{\gamma}_{i j}^{s}(0, \overrightarrow{0} ; \vec{k})\right] a_{s}(\vec{k})\right. \\
\left.+\left[\widetilde{\gamma}_{i j}^{s *}(\eta, \vec{x} ; \vec{k})-e^{-\rho k} \widetilde{\gamma}_{i j}^{s *}(0, \overrightarrow{0} ; \vec{k})\right] a_{s}^{\dagger}(\vec{k})\right\} .
\end{aligned}
$$


It is immediately apparent that the new mode functions are better behaved near $k=0$, so that the mode sum for the transformed Wightman function converges,

$$
\begin{aligned}
& W^{\prime}\left(x ; x^{\prime}\right)=\frac{1}{2} d(d-3) \\
& \quad \times \int d^{d-1} k\left[u(\eta, \vec{x} ; \vec{k})-e^{-\rho k} u(0, \overrightarrow{0} ; \vec{k})\right]\left[u^{*}\left(\eta^{\prime}, \vec{x}^{\prime} ; \vec{k}\right)-e^{-\rho k} u(0, \overrightarrow{0} ; \vec{k})\right] .
\end{aligned}
$$

Because the change from $h_{i j}(\eta, \vec{x})$ to $h_{i j}^{\prime}(\eta, \vec{x})$ was a gauge transformation, Higuchi, Marolf and Morrison conclude that no observable quantity is affected. Because Bunch-Davies vacuum now exists, they conclude that the graviton vacuum is de Sitter invariant.

Higuchi, Marolf and Morrison were aware of the dangers associated with employing a transformation parameter (32) which grows at spatial infinity. They therefore offered a local version of their argument in which the transformation parameter is made to vanish at spatial infinity [1]. The method consists of multiplying the old transformation parameter by a "tophat" function of space and time,

$$
\xi_{i}^{\text {new }}(\eta, \vec{x}) \equiv \xi_{i}^{\text {old }}(\eta, \vec{x}) \times T(\eta, \vec{x}) .
$$

The tophat function $T(\eta, \vec{x})$ is chosen to be unity within some arbitrarily large but finite region of space, and to vanish outside some larger but still finite region. Then the transformed graviton field agrees with (34) inside the smaller region, and the corresponding Wightman function is infrared finite within this region. Although the Wightman function diverges outside the larger region, they assert that this is unobservable because the smaller region can be made arbitrarily large. They even consider allowing the smaller region to encompass the past light-cone of some finite point back to $\eta \rightarrow-\infty$.

\section{Problems with the Transformation}

Let us first observe that the Wightman function of the transformed field has a simple interpretation in terms of the original Wightman function,

$$
\begin{array}{r}
W^{\prime}\left(x ; x^{\prime}\right)=W\left(x ; x^{\prime}\right)-\int d^{d-1} z F(\vec{z}, \rho) W(x ; 0, \vec{z})-\int d^{d-1} y F(\vec{y}, \rho) W\left(0, \vec{y} ; x^{\prime}\right) \\
\quad+\int d^{d-1} y F(\vec{y}, \rho) \int d^{d-1} z F(\vec{z}, \rho) W(0, \vec{y} ; 0, \vec{z})
\end{array}
$$


Here the real function $F(\vec{y}, \rho)$ is defined by Fourier transforming $e^{-\rho k}$,

$$
F(\vec{y}, \rho) \equiv \int \frac{d^{d-1} k}{(2 \pi)^{d-1}} e^{-i \vec{k} \cdot \vec{y}} e^{-\rho k}
$$

It is apparent that the transformed field is a sort of dipole with anti-sources at $\eta=0$ (which is the infinite future) to balance its sources at $x^{\mu}$ and $x^{\prime \mu}$. That is the origin of its better infrared behavior.

The problem with adding anti-sources is that it makes the transformed propagator obey a different equation from the original one even though no gauge condition has been changed. There are two plausible ways of defining what is meant by the transformed propagator, and neither of these definitions obeys (15). The simplest definition is just to extend (37),

$$
\begin{array}{r}
i \Delta_{1}^{\prime}\left(x ; x^{\prime}\right) \equiv i \Delta\left(x ; x^{\prime}\right)-\int d^{d-1} z F(\vec{z}, \rho) i \Delta(x ; 0, \vec{z})-\int d^{d-1} y F(\vec{y}, \rho) i \Delta\left(0, \vec{y} ; x^{\prime}\right) \\
\quad+\int d^{d-1} y F(\vec{y}, \rho) \int d^{d-1} z F(\vec{z}, \rho) i \Delta(0, \vec{y} ; 0, \vec{z}) .
\end{array}
$$

The equation obeyed by this propagator is,

$$
\sqrt{-g} \square i \Delta_{1}^{\prime}\left(x ; x^{\prime}\right)=\frac{1}{2} d(d-3)\left\{i \delta^{d}\left(x-x^{\prime}\right)-i \delta(\eta) F(\vec{x}, \rho)\right\} .
$$

The more orthodox definition for the transformed propagator is,

$$
i \Delta_{2}^{\prime}\left(x ; x^{\prime}\right) \equiv \theta\left(\eta-\eta^{\prime}\right) W^{\prime}\left(x ; x^{\prime}\right)+\theta\left(\eta^{\prime}-\eta\right) W^{\prime}\left(x^{\prime} ; x\right) .
$$

The equation it obeys is revealing,

$$
\begin{gathered}
\sqrt{-g} \square i \Delta_{2}^{\prime}\left(x ; x^{\prime}\right)=\left[a\left(\eta^{\prime}\right)\right]^{d-2}\left\{\delta\left(\eta-\eta^{\prime}\right)\left[\partial_{0} W^{\prime}\left(\eta^{\prime}, \vec{x}^{\prime} ; \eta, \vec{x}\right)-\partial_{0} W^{\prime}\left(\eta, \vec{x} ; \eta^{\prime}, \vec{x}^{\prime}\right)\right]\right. \\
\left.+\delta^{\prime}\left(\eta-\eta^{\prime}\right)\left[W^{\prime}\left(\eta^{\prime}, \vec{x}^{\prime} ; \eta^{\prime}, \vec{x}\right)-W^{\prime}\left(\eta^{\prime}, \vec{x} ; \eta^{\prime}, \vec{x}^{\prime}\right)\right]\right\}, \quad(42) \\
=\frac{1}{2} d(d-3) i \delta^{d}\left(x-x^{\prime}\right)+i\left[a\left(\eta^{\prime}\right)\right]^{d-2} \delta^{\prime}\left(\eta-\eta^{\prime}\right) \\
\times \int d^{d-1} y F(\vec{y}, \rho)\left[G\left(0, \vec{y} ; \eta^{\prime}, \vec{x}\right)-G\left(0, \vec{y} ; \eta^{\prime}, \vec{x}^{\prime}\right)\right] . \quad
\end{gathered}
$$

Higuchi, Marolf and Morrison checked that the equal-time commutator of the transformed field and its first time derivative on the first line of (42) is 
unchanged from (28) [1],

$$
\begin{aligned}
& {\left[a\left(\eta^{\prime}\right)\right]^{d-2} \delta\left(\eta-\eta^{\prime}\right)\left[\partial_{0} W^{\prime}\left(\eta^{\prime}, \vec{x}^{\prime} ; \eta, \vec{x}\right)-\partial_{0} W^{\prime}\left(\eta, \vec{x} ; \eta^{\prime}, \vec{x}^{\prime}\right)\right]} \\
& =\left[a\left(\eta^{\prime}\right)\right]^{d-2} \delta\left(\eta-\eta^{\prime}\right)\left[\partial_{0} W\left(\eta^{\prime}, \vec{x}^{\prime} ; \eta, \vec{x}\right)-\partial_{0} W\left(\eta, \vec{x} ; \eta^{\prime}, \vec{x}^{\prime}\right)\right] \\
& \quad=\frac{1}{2} d(d-3) i \delta^{d-1}\left(\vec{x}-\overrightarrow{x^{\prime}}\right)
\end{aligned}
$$

The extra term in (43) comes from the equal-time commutator of the undifferentiated fields. Higuchi, Marolf and Morrison seem to have assumed that this vanishes, just as its does for the original fields (27). That would be a safe assumption for a local transformation, but it is false for this nonlocal transformation. The source of the extra term in (43) is a change in the canonical commutation relations (27).

Additional evidence is provided by the retarded Green's function of the transformed field. If this is defined from the transformed Wightman function in the way one would normally do, assuming the canonical commutation relations (27-28), one finds that it changes,

$$
\begin{aligned}
G^{\prime}\left(x ; x^{\prime}\right) & \equiv-i \theta\left(\eta-\eta^{\prime}\right)\left[W^{\prime}\left(x ; x^{\prime}\right)-W^{\prime}\left(x^{\prime} ; x\right)\right] \\
& =G\left(x ; x^{\prime}\right)+\theta\left(\eta-\eta^{\prime}\right) \int d^{d-1} y F(\vec{y}, \rho)\left[G(0, \vec{y} ; x)-G\left(0, \vec{y} ; x^{\prime}\right)\right] .
\end{aligned}
$$

But the retarded Green's function follows from the unchanged field equations of classical general relativity and cannot change. Of course it does not change; the problem is that its relation to the Wightman function (46) assumes the canonical commutations relations (27,28), which are not correct for the transformed field.

The reason the canonical commutation relations changed is obvious in retrospect. The transverse-traceless-synchronous conditions (5) already fix the gauge completely; there is no additional local gauge freedom. One can see that from the fact that the field equation (6) and the canonical commutation relations (27) 28) give a unique solution (25). So any transformation which alters the mode sum without disturbing the transverse-traceless-synchronous condition (5) can only do so by changing the canonical commutation relations (27,-28).

Transformation (33) shifts the spacetime constant part of the field. This would not matter if the shift were by a $\mathbb{C}$-number but it matters a great deal when the shift is by a quantum operator because that operator possesses correlations with other operators whose spacetime dependence it can 
acquire. What (33) does is to load up the constant part of the field - which should have been an independent degree of freedom — with a superposition of creation and annihilation operators of all different wave numbers and spins. This becomes obvious if we rewrite the transformation parameter (32) in terms of the fields,

$$
\begin{aligned}
\xi_{i}(\eta, \vec{x}) & =-a^{2}(\eta) \int d^{d-1} y F(\vec{y}, \rho) \widetilde{h}_{i j}(0, \vec{y}) x^{j} \\
\widetilde{h}_{i j}^{\prime}(\eta, \vec{x}) & =\widetilde{h}_{i j}(\eta, \vec{x})-\int d^{d-1} y F(\vec{y}, \rho) \widetilde{h}_{i j}(0, \vec{y}) .
\end{aligned}
$$

From these expressions we see that violations of the propagator equation (15) and of the canonical commutation relations (27) occur in both the global version of the transformation (32) and also the localized version (36). This is significant because the localized version does change the gauge conditions in the transition region outside the smaller region and inside the larger region.

The altered commutation relations mean that Higuchi, Marolf and Morrison have potentially changed the physics of dynamical gravitons, rather than discovering a hitherto hidden property of the existing physics. The conclusion for the original theory is therefore unchanged: there is no de Sitter invariant state for dynamical gravitons. However, the willingness of mathematical physicists to resort to such ingenious expedients to salvage some form of de Sitter invariance for gravitons suggests that one should examine the viability of changing canonical commutation as has been done.

We can see three obvious problems. The first is that the new modes seem to modify the tensor power spectrum, 1

$$
\begin{aligned}
\Delta_{h}^{2}(k) & \equiv\left[32 \pi G(2 \pi)^{3}\right] \frac{k^{3}}{2 \pi^{2}} \int d^{3} x e^{-i \vec{k} \cdot \vec{x}}\left\langle\Omega\left|\widetilde{h}_{i j}^{\prime}(0, \vec{x}) \widetilde{h}_{i j}^{\prime}(0, \overrightarrow{0})\right| \Omega\right\rangle \\
& =\left[32 \pi G(2 \pi)^{3}\right] \frac{k^{3}}{2 \pi^{2}} \sum_{s} \widetilde{\gamma}_{i j}^{s}(0, \overrightarrow{0} ; \vec{k}) \widetilde{\gamma}_{i j}^{s *}(0, \overrightarrow{0} ; \vec{k}) \times\left[1-e^{-\rho k}\right], \\
& =\frac{16 G}{\pi \ell^{2}} \times\left[1-e^{-\rho k}\right] .
\end{aligned}
$$

The factor of $\left[1-e^{-\rho k}\right]$ multiplying the usual de Sitter result in expression (52) is more evidence that physics has been changed. We are not told the numerical value of $\rho$ relative to observable cosmological wave numbers $k$ but,

\footnotetext{
${ }^{1}$ The unfamiliar factor at the beginning of expression (50) arises from the way Higuchi, Marolf and Morrison define Fourier transforms and normalize the graviton field.
} 
if it is any natural scale of fundamental theory - from the Planck length to the Compton wavelength of a neutrino - then one has $\left[1-e^{-\rho k}\right] \approx \rho k$ for observable perturbations. That would give the graviton power spectrum a massive blue tilt, corresponding to a spectral index of $n_{T}=+1$. The usual prediction is $n_{T}$ negative and much less than one [23]; for single scalar inflation the data implies $-0.028<n_{T}<0$ at $95 \%$ confidence [4, 24].

The second problem is that changing canonical quantization could be done as well for the MMC scalar. Indeed, with the graviton indices contracted across index groups as we have been doing, the graviton relations are just $\frac{1}{2} d(d-3)$ copies of the MMC scalar relations. Making the same change in the scalar commutations relations would produce a propagator whose coincidence limit no longer agrees with the classic result of Vilenkin and Ford, Linde and Starobinsky [9], or with the results that follow from it when interactions are present [11, 12, 13]. These problems are confined to the realm of pure theory, whereas the ultimate justification of any physical model should be how it explains the data. Here the decisive fact seems to be that the scalar power spectrum would not be even approximately scale invariant as with the tensor power spectrum), in conflict with observation [24].

The final problem is that the very same type of gauge transformation changes the infrared behavior of the photon propagator in flat space QED. Consider free dynamical photons in Coulomb-synchronous gauge,

$$
A_{0}(t, \vec{x})=0 \quad, \quad \partial_{i} A_{i}(t, \vec{x})=0 .
$$

In $d=4$ spacetime dimensions the mode sum takes the form,

$$
A_{i}(t, \vec{x})=\int d^{3} k \sum_{s}\left\{\gamma_{i}^{s}(t, \vec{x} ; \vec{k}) a_{s}(\vec{k})+\gamma_{i}^{s *}(t, \vec{x} ; \vec{k}) a_{s}^{\dagger}(\vec{k})\right\},
$$

where the mode functions are,

$$
\gamma_{i}^{s}(t, \vec{x} ; \vec{k})=\frac{1}{\sqrt{(2 \pi)^{3} 2 k}} e^{-i k t+i \vec{k} \cdot \vec{x}} \epsilon_{i}^{s}(\vec{k}) .
$$

The polarization vectors $\epsilon_{i}^{s}(\vec{k})$ are the usual ones, and the creation and annihilation operators obey the same relations (24) as those for dynamical gravitons. In direct analogy to (48) we make a gauge transformation with parameter,

$$
\theta(t, \vec{x})=\int d^{3} y F(\vec{y}, \rho) A_{i}(0, \vec{y}) x^{i}
$$


Just as was the case with (32), the transformed vector potential continues to obey the Coulomb-synchronous conditions (53),

$$
A_{i}^{\prime}(t, \vec{x}) \equiv A_{i}(t, \vec{x})-\partial_{i} \theta(t, \vec{x})=A_{i}(t, \vec{x})-\int d^{3} y F(\vec{y}, \rho) A_{i}(0, \vec{y}) .
$$

As was the case with gravitons, the new photon mode sum is better behaved in the infrared,

$$
\begin{aligned}
A_{i}^{\prime}(t, \vec{x})=\int d^{3} k \sum_{s}\left\{\left[\gamma_{i}^{s}(t, \vec{x} ; \vec{k})\right.\right. & \left.-e^{-\rho k} \gamma_{i}^{s}(0, \overrightarrow{0} ; \vec{k})\right] a_{s}(\vec{k}) \\
& \left.\left.+\left[\gamma_{i}^{s *}(t, \vec{x} ; \vec{k})-e^{-\rho k} \gamma_{i}^{s *}(0, \overrightarrow{0} ; \vec{k})\right] a_{s}^{\dagger}(\vec{k})\right]\right\} .
\end{aligned}
$$

Indeed, it seems to be too well behaved. The old mode sum involved an integrable $1 / k$ divergence which allows the position-space propagator to be computed but leads to infrared divergences in loop corrections to exclusive scattering amplitudes. Those divergences are not some sort of gauge artifact, they arise from our inability to measure exclusive processes. When the problem is corrected by shifting to inclusive amplitudes the infrared behavior of the photon propagator leads to an observable dependence on the detector sensitivity that agrees with experiment [6]. None of this seems to be true for the new mode sum because it fails to diverge at $k=0$.

One might object that QED scattering amplitudes are gauge invariant, so they cannot be changed by making a gauge transformation. This argument is false because the gauge transformation was not made on the full, interacting theory. Indeed, it cannot be made on the full theory because the transformation exploited special properties of the free theory such as (53+54) that are no longer true in the presence of interactions. What is being done instead is to change the canonical commutation relations, and that generally does change physics. The problem is that it seems to change things we have already measured.

\section{Discussion}

As we have seen, what appears to be a linearized gauge transformation is actually a way of altering the canonical commutation relations (27) 28) of dynamical gravitons in transverse-traceless-synchronous gauge. This is because 
adding a $\mathbb{C}$-number constant is not the same as adding a constant operator. The correlations of the latter with other operators can permit it to inherit their spacetime dependence.

One consequence is that the theory of dynamical gravitons is being changed, rather than that some hidden property of the usual theory is being uncovered. The conclusion for the original theory is unambiguous: the Bunch-Davies wave function is not normalizable for any spatially flat cosmology with deceleration parameter $q \leq+\frac{1}{2}$. Because de Sitter (with $q=-1$ ) is just one of a wide range of cosmologies for which dynamical gravitons possess infrared problems, one should not opine that the resolution of its infrared problem would be ever so much clearer on the full de Sitter manifold. No such appeal is possible for cosmologies with $-1<q \leq+\frac{1}{2}$, so any argument about the infrared problem being a gauge artifact must make sense for spatially flat, FRW cosmologies with $q \leq+\frac{1}{2}$.

Is the new theory an object of interest? Many strange things happen when theories are not quantized canonically. Not all of these things are provably wrong but this particular procedure seems to be. It could be used to avoid the infrared divergence of the massless, minimally coupled scalar and seems to change both the tensor and scalar power spectra. For flat space QED the same sort of transformation seems to eliminate the infrared divergences of the exclusive scattering amplitudes. These things have measurable consequences and cannot be altered. The safest conclusion at this stage is that extraordinary claims require extraordinary proofs. Someone asserting that the new commutation relations give an acceptable theory (which no one has) should supply this proof, and that must include more than observing that the new relations were obtained by making what seems to be a gauge transformation.

Our comments apply to both the global version (32) and the localized version (36) of the transformation. However, it should be noted that the localized version of the transformation is not relevant to the discussion of de Sitter invariance. By definition, de Sitter invariance is a global property of the full state, not a local one, so no procedure which leaves the propagator divergent outside some arbitrary but large region could be invoked to establish it.

If it could be done, establishing the de Sitter invariance of dynamical gravitons would seriously alter how individual particles propagate through the vast ensemble of infrared gravitons which is created by inflation - at least in the usual physics. This problem has been studied at one loop order for massless fermions [26] and for MMC scalars [25]. The results are that 
MMC scalars suffer no significant correction whereas massless fermions experience a secular enhancement of their field strengths that eventually becomes nonperturbatively strong. The difference between the two particles seems to be spin; MMC scalars only interact with inflationary gravitons through their kinetic energies, which rapidly redshift to zero. Fermions experience an additional interaction due to spin, and it is precisely the diagrams involving the spin connection which are responsible for the one loop effect [27]. That suggests there should be a similarly strong effect for gravitons and photons. Neither computation has been made yet but the presence of infrared logarithms (which are not present in the altered theory of Higuchi, Marolf and Morrison [1]) in the one loop graviton self-energy argues that they also experience a strong effect [28].

Although we do not believe that even free dynamical gravitons are de Sitter invariant, it should be noted that their propagator does not give the full gravitational response to a source, nor even the most important part of this response. In a covariant gauge such as de Donder [20] the full propagator includes the effects of constraints as well as of dynamical gravitons. Of the classic tests of general relativity, all but the spin-down of the binary pulsar have to do with the constrained fields, not with the dynamical ones. This comment is especially significant for the quantum gravitational backreaction on inflation because the physical mechanism proposed for it [29] involves the gravitational response to the source provided by the continual inflationary production of dynamical gravitons. The stress-energy of these gravitons does not break de Sitter invariance at lowest order [30], any more than the stress-energy of the MMC scalar does. The lowest order secular back-reaction derives from the way the constrained fields respond to more and more inflationary gravitons appearing inside the past light-cone.

It should also be noted that even the manifest de Sitter invariance of the full graviton propagator (which no one seems to be maintaining any longer) would not preclude secular back-reaction from loop corrections. These loop corrections involve integrations of products of propagators and vertex operators. A de Sitter invariant propagator just means that the integrands are de Sitter invariant; the result of performing the integrals can still break de Sitter invariance [31. A classic example is the function $\theta\left(-\ell^{2}\left(x ; x^{\prime}\right)\right)$, where $\ell\left(x ; x^{\prime}\right)$ is the invariant distance from $x^{\mu}$ to $x^{\prime \mu}$. This function is certainly invariant: it is one inside the light-cone and zero outside. Yet its integral over $x^{\prime \mu}$ to the past of $x^{\mu}$, back to the initial value surface, gives the invariant volume of the past light-cone. That quantity grows as $x^{\mu}$ is evolved to the 
future. This example is not specious. Precisely this theta function appears as the "tail term" of the retarded graviton propagator (17).

\section{Acknowledgements}

We are grateful to D. Marolf for his courtesy in bringing an early version of his paper to our attention, and for patiently answering our questions and comments over the course of many e-mail exchanges. We also thank S. Deser , D. Marolf and I. Morrison for reading a preliminary version of this paper and providing valuable comments. This work was partially supported by NWO Veni Project \# 680-47-406, by European Union Grant FP-7-REGPOT-20081-CreteHEPCosmo-228644, by NSF grant PHY-0855021, and by the Institute for Fundamental Theory at the University of Florida.

\section{References}

[1] A. Higuchi, D. Marolf and I. A. Morrison, arXiv:1107.2712.

[2] A. A. Starobinsky, JETP Lett. 30 (1979) 682; V. F. Mukhanov and G. V. Chibisov, JETP Lett. 33 (1981) 532.

[3] T. S. Bunch and P. C. W. Davies, Proc. R. Soc. A357 (1977) 381.

[4] E. O. Kahya, V. K. Onemli and R. P. Woodard, Phys. Lett. B694 (2010) 101, arXiv:1006.3999.

[5] L. H. Ford and L. Parker, Phys. Rev. D16 (1977) 1601.

[6] F. Bloch and H. Nordsieck, Phys. Rev. 52 (1937) 54; S. Weinberg, Phys. Rev. bf 140 (1965) B516.

[7] A. Vilenkin, Nucl. Phys. B226 (1983) 527; N. C. Tsamis and R. P. Woodard, Class. Quant. Grav. 11 (1994) 2969.

[8] T. M. Janssen, S. P. Miao, T. Prokopec and R. P. Woodard, Class. Quant. Grav. 25 (2008) 245013, arXiv:0808.2449; JCAP 0905 (2009) 003, arXiv:0904.1151.

[9] A. Vilenkin and L. H. Ford, Phys. Rev. D26 (1982) 1231; A. D. Linde, Phys. Lett. 116B (1982) 335; A. A. Starobinsky, Phys. Lett. 117B (1982) 175. 
[10] B. Allen and A. Folacci, Phys. Rev. D35 (1987) 3771.

[11] A. A. Starobinsky and J. Yokoyama, Phys. Rev. D50 (1994) 6357, astro-ph/9407016; V. K. Onemli and R. P. Woodard, Class. Quant. Grav. 19 (2002) 4607, qc-gr/0204065; Phys. Rev. D70 (2004) 107301, gr-qc/0406098; T. Brunier, V. K. Onemli and R. P. Woodard, Class. Quant. Grav. 22 (2005) 59, gr-qc/0408080; E. O. Kahya and V. K. Onemli, Phys. Rev. D76 (2007) 043512, gr-qc/0612026.

[12] T. Prokopec and R. P. Woodard, JHEP 0310 (2003) 059, astro-ph/0309593; B. Garbrecht and T. Prokopec, Phys. Rev. D73 (2006) 064036, gr-qc/0602011; S. P. Miao and R. P. Woodard, Phys. Rev. D74 (2006) 044019, gr-qc/0602110.

[13] T. Prokopec, O. Tornkvist and R. P. Woodard, Phys. Rev. Lett. 89 (2002) 101301, astro-ph/0205331; Annals Phys. 303 (2003) 251, gr-qc/0205130; T. Prokopec and R. P. Woodard, Annals Phys. 312 (2004) 1, gr-qc/0310056; T. Prokopec, N. C. Tsamis and R. P. Woodard, Class. Quant. Grav. 24 (2007) 201, gr-qc/0607094; Annals Phys. 323 (2008) 1324, arXiv:0707.0847; Phys. Rev. D78 (2008) 043523, arXiv:0802.3673.

[14] L. P. Grishchuk, Sov. Phys. JETP 40 (1975) 409.

[15] N. C. Tsamis and R. P. Woodard, Phys. Lett. B292 (1992) 269; S. Deser and A. Waldron, Phys. Lett. B513 (2001) 137, hep-th/0105181; Nucl. Phys. B662 (2003) 379, hep-th/0301068.

[16] N. C. Tsamis and R. P. Woodard, Commun. Math. Phys. 162 (1994) 217.

[17] B. Allen and M. Turyn, Nucl. Phys. B292 (1987) 813; S. W. Hawking, T. Hertog and N. Turok, Phys. Rev. D62 (2000) 063502, hep-th/0003016; A. Higuchi and S. S. Kouris, Class. Quant. Grav. 18 (2001) 4317, gr-qc/0107036; A. Higuchi and R. H. Weeks, Class. Quant. Grav. 20 (2003) 3006, gr-qc/0212031; A. Higuchi and Y. C. Lee, Phys. Rev. D78 (2008) 084031, arXiv:0808.0642; M. Faizal and A. Higuchi, Phys. Rev. D78 (2008) 067502, arXiv:0806.3735.

[18] S. P. Miao, N. C. Tsamis and R. P. Woodard, J. Math. Phys. 50 (2009) 122502, arXiv:0907.4930. 
[19] S. P. Miao, N. C. Tsamis and R. P. Woodard, J. Math. Phys. 51 072503, arXiv:1002.4037.

[20] S. P. Miao, N. C. Tsamis and R. P. Woodard, arXiv:1106.0925.

[21] A. Higuchi, Class. Quant. Grav. 8 (1991) 1983; Class. Quantu. Grav. 8 (1991) 2005.

[22] D. Marolf and I. A. Morrison, Class. Quant. Grav. 26 (2009) 235003, arXiv:0810.5163, M. Faizal and A. Higuchi, arXiv:1107.0395.

[23] V. F. Mukhanov, H. A. Feldman and R. H. Brandenberger, Phys. Rep. 215 (1992) 203; A. R. Liddle and D. H. Lyth, Phys. Rep. 231 (1993) 1, astro-ph/9393919 J. E. Lidsey, A. R. Liddle, E. W. Kolb, E. J. Copeland, T. Barreiro and M. Abney, Rev. Mod. Phys. 69 (1997) 373, astro-ph/9508078.

[24] E. Komatsu et al., Astrophys. J. Suppl. 192 (2011) 18, arXiv:1001.4538.

[25] E. O. Kahya and R. P. Woodard, Phys. Rev. D76 (2007) 124005, arXiv:0709.0536; Phys. Rev. D77 (2008) 084012, arXiv:0710.581.

[26] S. P. Miao and R. P. Woodard, Class. Quant. Grav. 23 (2006) 1721, gr-qc/0511140; Phys. Rev. D74 (2006) 024021, gr-qc/0603135; S. P. Miao, arXiv:0705.0767.

[27] S. P. Miao and R. P. Woodard, Class. Quant. Grav. 25 (2008) 145009, arXiv:0803.2377.

[28] N. C. Tsamis and R. P. Woodard, Phys. Rev. D54 (1996) 2621, hep-ph/9602317.

[29] N. C. Tsamis and R. P. Woodard, Phys. Lett. B301 (1993) 351; Nucl. Phys. B474 1996) 235, hep-ph/9602315; Ann. Phys. 253 (1997) 1, hep-ph/9602316; Ann. Phys. 267 (1998) 145, hep-ph/9712331; arXiv:1103.5134.

[30] N. C. Tsamis and R. P. Woodard, Ann. Phys. 321 (2006) 875, gr-qc/0506056.

[31] R. P. Woodard, gr-qc/0408002. 